$10-2000$

\title{
The Effects of Patch Properties on the Debonding Behavior of Patched Beam-Plates
}

Anette M. Karlsson

Cleveland State University, a.karlsson@csuohio.edu

Follow this and additional works at: https://engagedscholarship.csuohio.edu/enme_facpub

Part of the Materials Science and Engineering Commons, and the Mechanical Engineering Commons How does access to this work benefit you? Let us know!

\section{Original Citation}

Karlsson, A. M., 2000, "The Effects of Patch Properties on the Debonding Behavior of Patched BeamPlates," Journal of Engineering Materials and Technology, 122(4) pp. 389-393.

This Article is brought to you for free and open access by the Mechanical Engineering Department at EngagedScholarship@CSU. It has been accepted for inclusion in Mechanical Engineering Faculty Publications by an authorized administrator of EngagedScholarship@CSU. For more information, please contact library.es@csuohio.edu. 


\section{The Effects of Patch Properties on the Debonding Behavior of Patched Beam-Plates}

The debonding characteristics of patched structures are investigated in this study by means of an analytical model. In particular, the effects the lay-up sequence and edgetapering of a carbon-reinforced epoxy patch, as well as the beveling of an aluminum patch, have on the initiation, stability, and extent of the debonding are considered. The results presented show that both the degree of edge-tapering and the patch properties must be carefully selected in order to optimize the patched structure. It is also shown that when designing a patched system, it is important to model the correct boundary and load conditions to correctly simulate the debonding behavior.

\section{Introduction}

Patched structures is a common engineering design, consisting of a secondary structure attached to a base structure, with the purpose of strengthening, stiffening, or providing thermal or electrical contact or insulation. This type of structures can be found in a range of applications, for example, as repair-patches on aircraft structures where the patches are used to prevent existing cracks from growing. In this case, it is of course of primary interest to establish if a patch will prevent a crack from growing, and studies have indicated that adhering a patch over a crack is indeed a very efficient way of preventing further crack growth (for example Baker [1], Chiu et al. [2], Chue et al. [3], Park et al. [4], Paul and Jones [5], Rodrick [6], Sih and Hong [7], and Tarn and Shek [8]). However, it is also of significance to determine the integrity of the structure: the patch may not, for obvious reasons, be allowed to disintegrate from the base structure. The debonding behavior of structures repaired with patches will be the topic of this paper.

By adhering a patch to an exciting structure, an anisotropic composite structure is created, including a jump in the neutral axis and a change in the (local) stiffness. The geometric and constitutive discontinuity causes a range of interesting behavior, including the debonding of the patch from the base structure and a diversity of thermally induced deflection modes. Initial investigations of the thermally induced behavior was recently conducted by Karlsson and Bottega [9-11]. However, presently we will limit the discussion to mechanical loading (no thermal loading) and discuss some aspects of the failure of patched beam-plates with respect to edge debonding of the patch from the base structure.

In previous studies relating to the debonding of patched structures (Bottega [12], Bottega and Karlsson [13], Karlsson and Bottega $[14,15]$ and Loia and Bottega [16]) analytical and qualitative investigations were conducted. It was seen that a change in loading or support conditions significantly changes the characteristics of the debonding and that the unbonded part of the patched has to be included in the analysis. Other important results showed that relative compliant and relative long patches were preferred. Commonly, the edges of the patch are beveled, or tapered, to reduce the stress concentration the discontinuity associated with the patch introduces. However, Karlsson and Bottega [15] showed that the taper angle has to be carefully selected since there may be intermediate regions of taper angles that promote debonding rather than prevent it.

Since the investigations discussed in the previous paragraph were general in their objectives, i.e., they applied to any type of patched structures and the results were presented in a normalized manner, it is of interest to investigate some actual structures that relates to aerospace structures. In this study an analytical investigation will be conducted where the base structure under consideration is a flat aluminum plate and the patch is made of either aluminum or carbon reinforced epoxy. The parameters considered are: various taper angles, various lay-up sequences, and a range of support geometries and loading conditions. The results will be discussed in terms of the initiation, extent and stability of the debonding process.

\section{Problem Formulation and Analytical Solutions}

The analytical model used in this study was developed by Bottega and Karlsson [13]. In this model, the half-span of the base structure (normalized length $L_{0}$ ) is considered and any number of layers in the patch is allowed. The taper angle is defined so that $\psi=0$ deg corresponds to an untapered patch, see Fig. 1. The patch of length $L_{1}$ has a perfect bond (by adhesion) to the base structure over the region $0<s \leqslant a$, where $0<a \leqslant L_{1}$. It was shown by Bottega and Karlsson [13] that the unbonded part of the patch may be in either one of three configurations: (i) full contact: the unbonded region of the patch remains in full sliding contact with the base structure, (ii) edge point contact: the unbonded region of the patch has lifted up from the base structure, except the edge of the patch, which remains in sliding contact with the base structure, (iii) no contact: the unbonded part of the patch has totally lifted off the base structure. The conjugate bond zone size is defined as $a^{*}$ $=L_{0}-a$, with which the propagating bond zone boundary will be monitored. The transverse deflections, $w$, are measured positive downwards, and the in-plane deflections, $u$, are positive in the direction of increasing $s$.

The analytical formulation of the problem is summarized in the following. The von Karman plate theory is used to model the individual layers in the patch and the base structure and a Griffith type fracture criteria is incorporated. The energy functional is formulated, consisting of: the bending and membrane energy for the individual layers in the patch and base structure; the constraint functional between the individual components; the work done by the applied loading; and the delamination energy. With this established, the principal of stationary potential energy is applied within the context of the problem. Taking the appropriate variations, allowing the interior boundaries to vary in order to simulate the propagating debonding, results in a set of nonlinear differential equations, boundary conditions for the edge of the base structure, matching conditions over the discontinuity of the patch and the step tapering within the patch, and the transversality condition. The transversality condition is the condition that establishes 


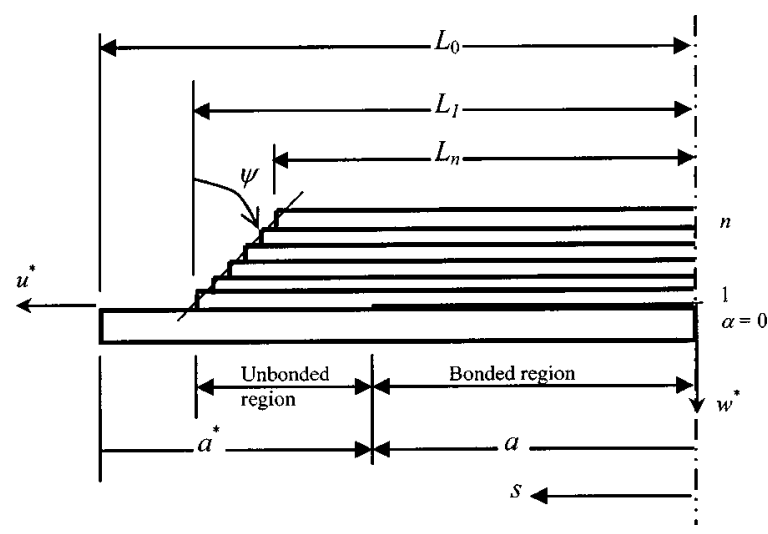

Fig. 1 Geometry of step tapered patch on base structure

the location of the propagating bond zone boundary associated with the equilibrium configurations. This condition will be discussed in further details below. A linear analytical solution is found by recasting the problem into "mixed formulation" where, instead of expressing the problem in terms of the two variables transverse and in-plane deflection, the problem is expressed in terms of transverse deflection and membrane force. The full problem formulation will not be repeated in this text for brevity, and the interested reader is referred to Bottega and Karlsson [13] for the details.

The transversality condition is the condition that establishes the location of the propagating contact zone associated with equilibrium configurations. It is found to be (Bottega and Karlsson [13])

Full Contact Zone:

$$
\mathcal{G}_{A}\{a\} \equiv\left[\frac{1}{2} \mu_{(q)}^{*} \kappa_{2}^{2}-\frac{1}{2} D_{(q)}^{*} \kappa_{1}^{2}+\left(\frac{1}{2 C_{0}}-\frac{1}{2 C_{(q)}^{*}}\right) N_{0}^{2}\right]_{s=a}=2 \gamma,
$$

\section{Edge Contact or No Contact:}

$$
\mathcal{G}_{B}\{a\} \equiv\left[\frac{1}{2} D_{0} \kappa_{03}^{2}-\frac{1}{2} D_{(q)}^{*} \kappa_{1}^{2}+\left(\frac{1}{2 C_{0}}-\frac{1}{2 C_{(q)}^{*}}\right) N_{0}^{2}\right]_{s=a}=2 \gamma
$$

where $D_{0}$ and $C_{0}$ are the normalized bending and membrane stiffnesses of the base structure with respect to its neutral axis, respectively, $\mu_{(q)}^{*}$ is the normalized bending stiffness of the composite structure with respect to the neutral axis in the contact zone, and $D_{(q)}^{*}$ and $C_{(q)}^{*}$ are the normalized bending and membrane stiffnesses of the composite structure with respect to the neutral axis in the bonded region, respectively. Subscript $q$ indicates the number of layers of the patch at $a . \kappa_{1}, \kappa_{2}$, and $\kappa_{03}$ are the curvature change in the bonded region, the contact region, and the unbonded region, respectively. $N_{0}$ is the normalized in-plane membrane force. Furthermore, $\gamma$ is the normalized bond energy of the adhesive bond between the patch and the base structure. All length scales are normalized with respect to the length of the bases structure, and the bond energy and membrane force are normalized by $\gamma=\bar{\gamma} \bar{L}^{2} / \bar{D}_{0}$ and $N_{0}=\bar{N}_{0} \bar{L}^{2} / \bar{D}_{0}$, respectively, where over-score indicates the dimensional counterparts.

In Eqs. (1) and (2), $\mathcal{G}_{A}\{a\}$ and $\mathcal{G}_{B}\{a\}$ are identified as the energy release rates. The conditions (1) and (2) suggest the following delamination criterion:

if, for some initial value of $a=a_{0}$, we have that $\mathcal{G}\left\{a_{0}\right\} \geqslant 2 \gamma$, then debonding occurs and the system evolves ( $a$ decreases, $a^{*}$ increases) such that the corresponding equality (1) or (2) is satisfied. If $\mathcal{G}\left\{a_{0}\right\}<2 \gamma$, debonding does not occur.

In addition to the set of equations and conditions discussed above, one additional condition is needed to fully describe the problem. By integrating the strain-displacement relations and im-

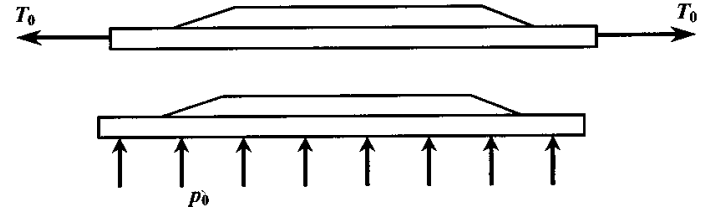

Fig. 2 Structure loaded with (a) applied in-plane tension, (b) applied transverse pressure

posing the corresponding boundary and matching conditions for the in-plane displacement, the integrability condition results, which in its linearized form is given by

$$
u_{03}\left(L_{0}\right)=\lambda \mathcal{F}_{\lambda}(a)+N_{0} \mathcal{F}_{N_{0}}(a)
$$

where $u_{03}\left(L_{0}\right)$ is the in-plane displacement at the edge of the base structure, $\lambda$ is the loading parameter, and $\mathcal{F}_{\lambda}$ and $\mathcal{F}_{N_{0}}$ are nonlinear function (see Bottega and Karlsson [13]). In the present case, the loading parameter simply corresponds to the normalized external load which is either a normalized in-plane tensile force, $\lambda$ $=T_{0}$, applied at the edge of the base structure, or a normalized transverse pressure, $\lambda=p_{0}$, applied on the base structure, see Fig. 2. $T_{0}$ and $p_{0}$ are related to their dimensional counterparts as $T_{0}$ $=\bar{T}_{0} \bar{L}^{2} / \bar{D}_{0}$ and $p_{0}=\bar{p}_{0} \bar{L}^{3} / \bar{D}_{0}$, respectively, where over-score indicates the dimensional counterparts. When the edges of the base structure are allowed to move in the plane, condition (3) gives the magnitude of the displacement for a given external load. For the case when the edges of the base structure are prevented from motion, $u_{03}\left(L_{0}\right)=0$, the corresponding membrane force $N_{0}$ may be solved from the expression (3).

It was proved by Bottega and Karlsson [13] that, within the context of the model, no contact zone or edge contact is present for the case of applied in-plane tension or for the case of applied pressure when the base structure is supported such that its edges are free to rotate (hinged supports). For the case of applied transverse pressure with edges prohibited from rotation (clamped supports), it was established that the presence of a full contact zone or edge contact is possible. When multiple solutions are possible, the one associated with the lowest total energy will be taken as the preferred configuration. It was shown by Karlsson and Bottega [15] that when multiple configurations are possible, the hierarchy of "preference" of the system is (1) full contact zone, (2) edgepoint contact, (3) no contact.

With the above established, the energy release rate can be written in terms of the loading parameter explicitly, for each case under consideration. This is so, since we are only concerned with the linear solution. ${ }^{1}$ The equations for the growth paths, the threshold curves, may be found directly from the transversality conditions (1) and (2), and take the general form

$$
T^{*} \equiv T_{0} / \sqrt{2 \gamma}=1 / \sqrt{\Omega\left(a^{*} ; \mathbf{S}\right)} \text { or } \quad p^{*} \equiv p_{0} / \sqrt{2 \gamma}=1 / \sqrt{\Omega\left(a^{*} ; \mathbf{S}\right)}
$$

where $\Omega\left(a^{*} ; \mathbf{S}\right)$ is the normalized energy release rate per square of the normalized load, $\mathbf{S}$ is the set of stiffnesses of the structure, and $T^{*}$ and $p^{*}$ are the renormalized critical loads, i.e., the load at which debonding is initiated.

\section{Results}

The specific structures investigated consist of a $100 \mathrm{~mm}$ long aluminum base structure of thickness $1 \mathrm{~mm}$ (thus the half span

\footnotetext{
${ }^{1}$ We note here that in general, interfacial fracture is a mixed mode problem. However, it was shown by Hutchinson and Suo [17] that in this class of problems, mode II is the predominant mode for sufficiently long cracks. Furthermore, it was seen in Karlsson and Bottega [15] that for the current combination of loading and geometry, the normal stresses are negative (thus crack closing in mode I) and mode I fracture cannot occur. It follows that the analysis and associated numerical results are valid for any initial size of the debond zone, even for $a^{*} \rightarrow 0$ (within the limitations of classic fracture mechanics).
} 
Table 1 Materials properties of aluminum and unidirection carbon fiber reinforced epoxy

\begin{tabular}{|c|c|c|}
\hline Aluminum & $E=72 \mathrm{GPa}$ & $v=0.33$ \\
\hline \multicolumn{3}{|c|}{ Graphite/epoxy } \\
\hline & Longitudinal Modulus & $128 \mathrm{GPa}$ \\
\hline & Transverse Modulus & $11.3 \mathrm{GPa}$ \\
\hline & Major Poisson's ratio & 0.3 \\
\hline & Shear Modulus & $6.0 \mathrm{GPa}$ \\
\hline & Ply thickness & $0.14 \mathrm{~mm}$ \\
\hline
\end{tabular}

$\bar{L}_{0}=50 \mathrm{~mm}$ ). The patch has the same length as the base structure and is made of either $1-\mathrm{mm}$ thick aluminum or, alternatively, 8 layers of carbon reinforce epoxy. Table 1 displays the associated material properties. In the following, the results are presented in terms of "threshold curves," as defined in Eq. (4), where the threshold curves displays the critical load (external load for which debonding is initiated) as a function of the conjugate bonds zone, $a^{*}=\bar{a}^{*} / \bar{L}$.

Hinged Edges and Applied In-Plane Tensile Force. For a structure where the edges of the base plate are hinged and loaded with an in-plane tensile force, the critical membrane force, $T^{*}$, is constant, see Fig. 3. A constant critical force corresponds to a load-independence of the initial unbonded region, and to a critical stable debonding once the critical force is achieved. By beveling the aluminum patch, the critical load increases but only within the

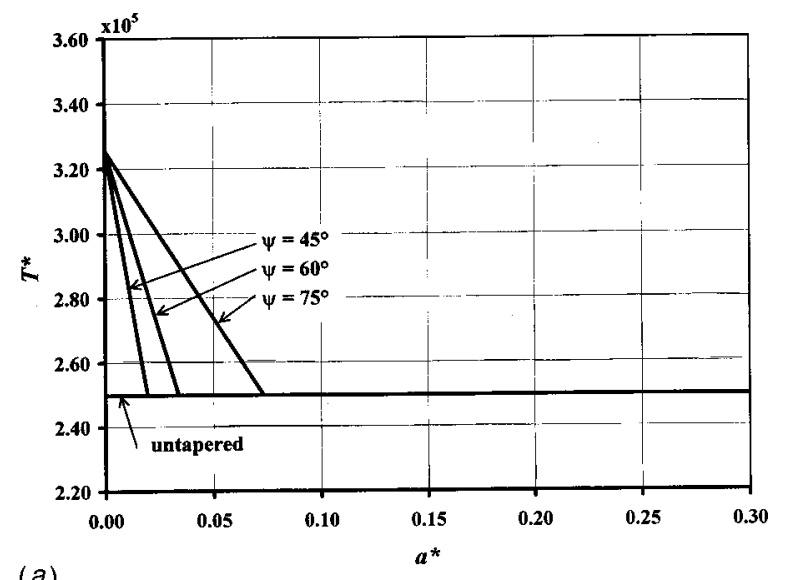

(a)

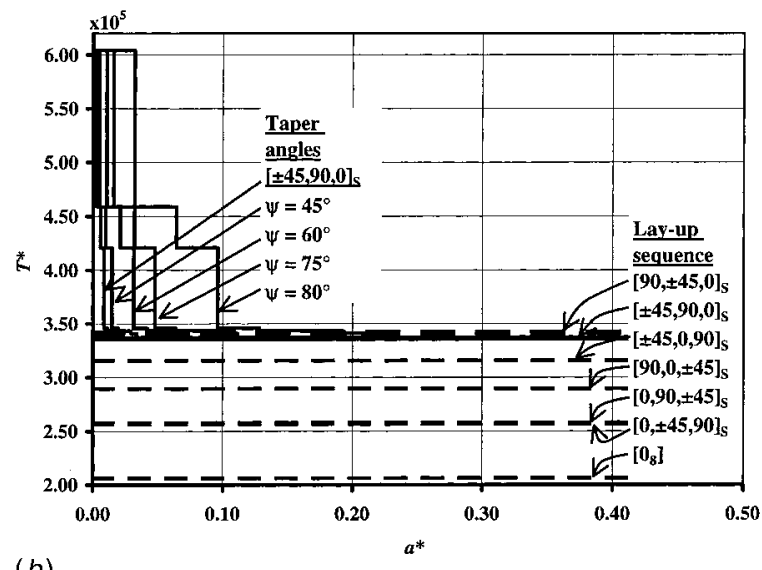

(b)

Fig. 3 Critical normalized tensile force, $T^{*}$, as a function of the normalized conjugate bond zone $a^{*}$ for tensile loaded structure with hinged edges. (a) Aluminum patch, (b) composite patch.

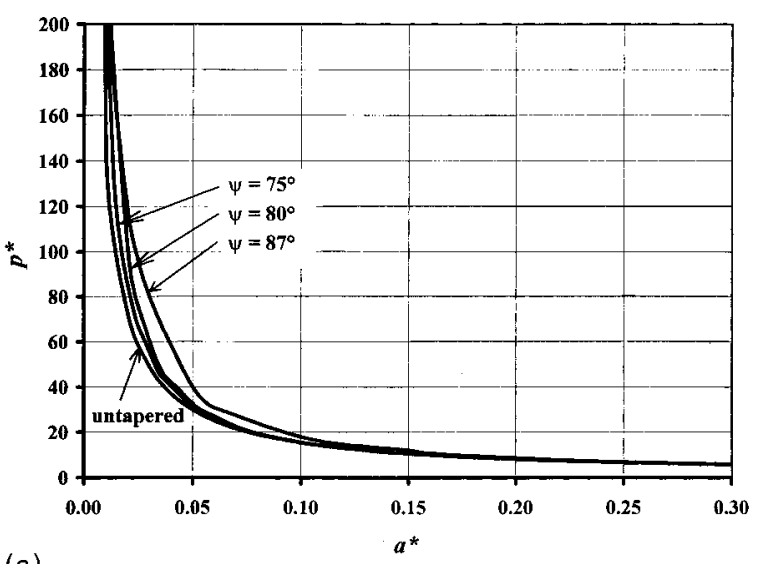

(a)

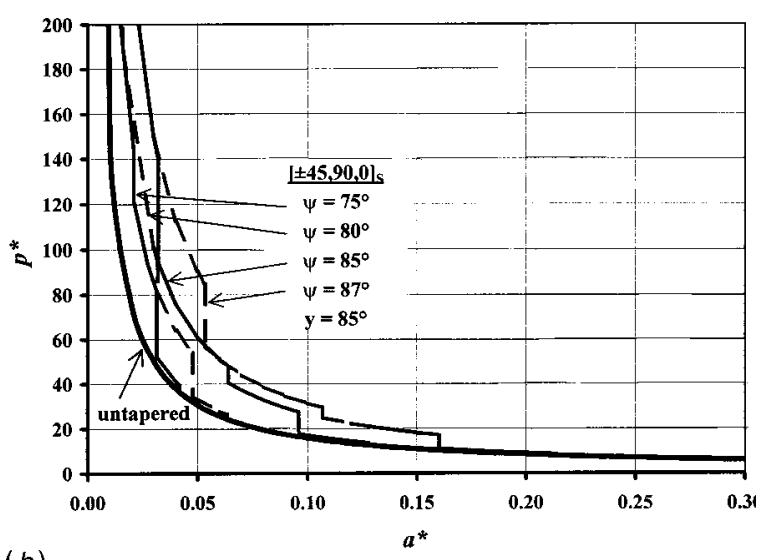

(b)

Fig. 4 Critical normalized transverse pressure, $p^{*}$, as a function of the normalized conjugate bond zone $a^{*}$ for pressure loaded structure with hinged-free edges. (a) Aluminum patch, (b) composite patch.

beveled region: once outside the beveled region, the threshold curves converge to the unbeveled case (Fig. 3(a)). In the region of increased threshold values, the debonding will be unstable once debonding occurs. Similar results are seen for the carbon reinforced composite patch in Fig. 3(b) (the discontinuities in the curves arise from the discontinuity in the step-tapering of the patch). It may be noted that tapering of the composite patch gives a significant increase in threshold values compared to the influence beveling of the aluminum patch has. Furthermore, by optimizing the stacking sequence of the individual layers in the composite patch the threshold value may be increased. $(0 \mathrm{deg}$ is in the direction of the load.) Lowest critical load is achieved for the unidirectional patch.

Note that the critical values in Fig. 3(a) and Fig. 3(b) cannot directly be compared, since the absolute value will be dependent on the bond strength, $2 \gamma$, between the patch and the base structure. Obviously, the bond strength will depend not only on the adhesive but also on the materials involved.

Hinged-Free Edges and Applied Transverse Pressure. The next case we will consider has the same boundaries as the previous case, i.e., hinged-free (hinged, to allow for rotation, and free, to allow for in-plane motion), but the structure is now subjected to a transverse pressure (Fig. 2(b)). In this case, the threshold curves no longer yield constant critical loads, but an asymptote to the line $a^{*}=0$ (Fig. 4). Thus, within the context of the model, a perfectly bonded patch will not start to debond. For small initial unbonded regions, debonding will occur when the critical pressure is reached and once initiated the debonding will be unstable. Bevel- 
ing an aluminum patch (Fig. 4(a)) and tapering a composite patch (Fig. 4(b)) yields higher threshold values within the region of beveling/tapering. The lay-up sequence does not (within the resolution of the figures) affect the critical pressure (not shown).

Clamped-Fixed Edges and Transverse Pressure. If the edges of the base structure are clamped from rotation and fixed to prevent in-plane deflections, (clamped-fixed edges) and the structure is subjected to a transverse pressure, the debonding characteristics changes significantly from the previous cases considered,

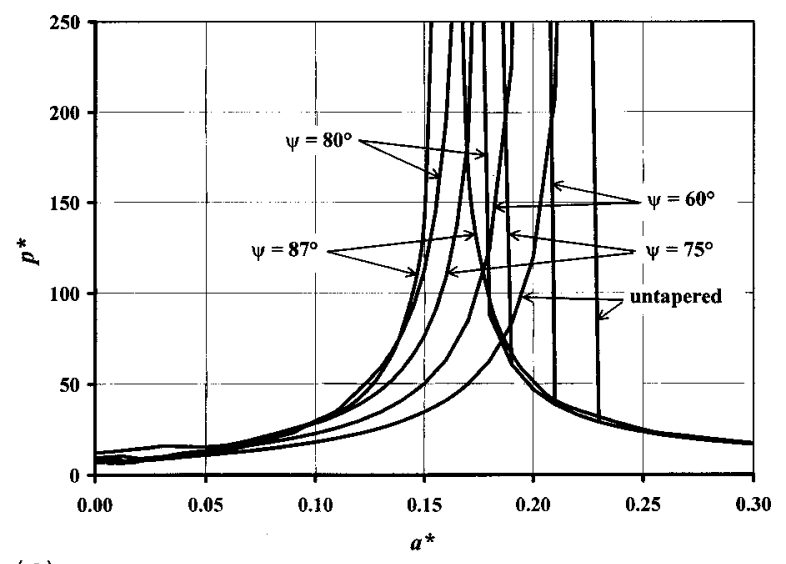

(a)

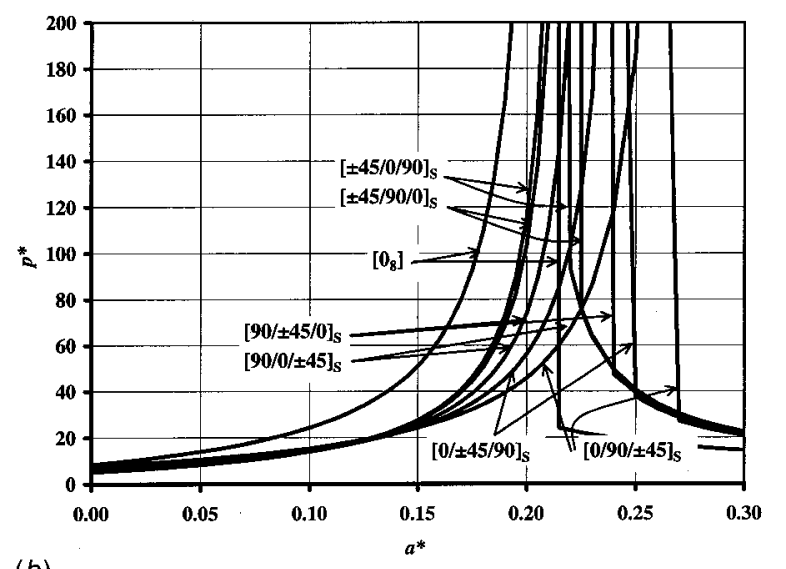

(b)

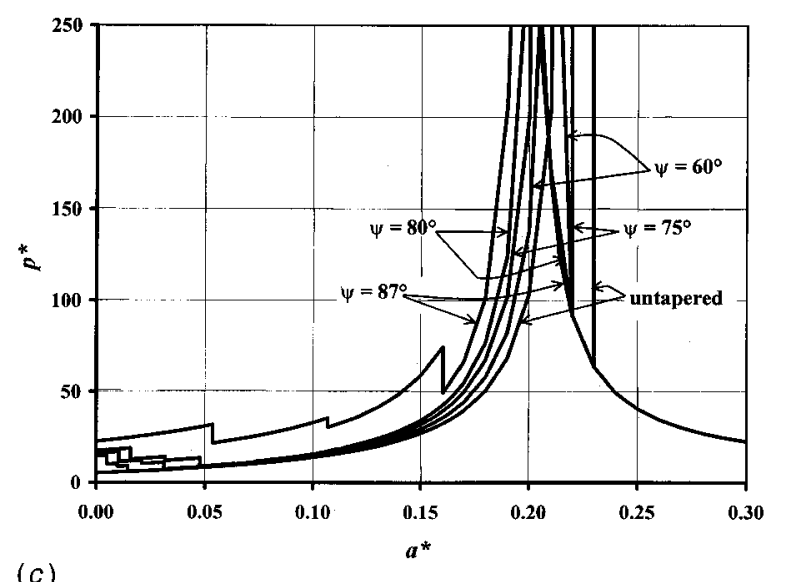

(c)

Fig. 5 Critical normalized transverse pressure, $p^{*}$, as a function of the normalized conjugate bond zone $a^{*}$ for pressure loaded structure with clamped-fixed edges. (a) Aluminum patch, (b) composite patch: various lay-up sequences, $(c)$ composite patch: various taper angles. including an asymptote dividing the path into two regions, Fig. 5. We recall that for this case, the debonded part of the patch may be in either "full contact," "edge point contact" or be completely lifted off from the base structure, as discussed in Section 2. In Fig. 5 , the "preferred" configurations are presented according to the ranking discussed in Section 2. Typically the transition from "full contact zone" to edge point contact occur at the right most discontinuity observed in the graphs, which, for most cases, occur in the right hand branch of the asymptote. The transition from edge point contact to "no contact"' is smooth.

For small initial unbonded regions ("left side" of the asymptote), the debonding will be stable once the critical pressure is reached. The debonding will effectively come to an arrest as the asymptote is approached. For larger initial unbonded regions ("right side" of the asymptote) the debonding is unstable once the critical pressure is reached. If the aluminum patch is beveled (Fig. 5(a)) or the composite patch tapered (Fig. 5(c)), the asymptote moves "left," toward lower values of the conjugate bond zone. Thus, if the patch has a small, initial unbonded region, the debonding will come to a stop "faster" as the degree of beveling/ tapering is increased. However, it follows that relative smaller defects can be tolerated, since the region of stable debonding has decreased. The location of the asymptote may be tailored by changing the lay-up sequence (Fig. 5(b)). By comparing the location of the asymptotes between the case of aluminum patch and composite patch, it may be seen that the asymptotes for composite patches occur at a higher value of the conjugate bond zone. Which scenario is preferred has to be determined for the specific application in question. Even though it may be attractive to make the debonding come to an arrest as soon as possible, it may also be of interest to allow for a larger initial unbonded region before stopping the debonding process, particularly when considering the various loading conditions an airplane structure is subjected to.

\section{Summary}

The debonding characteristics of a range of patched structures have been examined, based on an analytical model. In particular, a thin aluminum base structure with either a composite or an aluminum patch was investigated, where various support and loading conditions, various taper angles, and, for the composite patch, various lay-up sequences were considered.

The debonding characteristics are found to strongly depend, not only on the type of external loads the structure is subjected to, but also on the type of support conditions at the edge of the base structure. In order to design a patched structure, it is therefore important to establish both the various loading conditions and the type of boundary conditions the actual structure is subjected to. It may also be noted that few applications consist of the "extreme" boundaries used in this study and the "true" support conditions will be somewhere between "hinged-free" and "clamped-fixed."

In this study, it is seen that the debonding characteristics may be optimized for a structure by an appropriate selection of the properties of the patch. For example, by selecting a compliant layer closest to the base structure, the patch becomes the least prone for debonding. It was also seen in the study that the commonly used unidirectional repair patch has in many cases the least desired debonding characteristics, and should be used with caution. In addition the taper/beveling angle strongly influences the debonding behavior, and for clamped-fixed boundary conditions may be selected to optimize the maximum allowable unbonded region.

\section{References}

[1] Baker, A. A., 1993, "Repair Efficiency in Fatigue-Cracked Aluminum Components Reinforced With Boron/Epoxy Patches," Fatigue Fract. Eng. Mater. Struct., 66, No. 7, pp. 753-765.

[2] Chiu, W. K., Rees, D., Chalkey, P., and Jones, R., 1994, "Designing for Damage-Tolerant Composite Repairs,' Comp. Struct., 28, pp. 19-37.

[3] Chue, C.-H., Chang, L.-C., and Tsai, J.-S., 1994, "Bonded Repair of Plate 
With Inclined Central Crack Under Biaxial Loading," Comp. Struct., 28, pp. $39-45$.

[4] Park, J. H., Ogiso, T., and Atluri, S. N., 1992, "Analysis of Cracks in Aging Aircraft Structures, With and Without Composite-Patch Repairs," Comp. Mech., 10, No. 10, pp. 169-201.

[5] Paul, J., and Jones, R., 1992, “Repair of Impact Damaged Composites,' Eng. Fract. Mech., 41, No. 1, pp. 127-141.

[6] Roderick, G. L., 1980, "Prediction of Cyclic Growth of Cracks and Debonds on Aluminum Sheets Reinforced With Boron/Epoxy," Fibrous Composites in Structural Design, E. M. Lenoe, D. W. Oplinger, J. J. Burke, eds., Plenum Press, New York, pp. 467-481.

[7] Sih, G. C., and Hong, T. B., 1989, "Integrity of Edge-Debonded Patch on Cracked Panel," Theor. Appl. Fract. Mech., 12, pp. 121-143.

[8] Tarn, J.-Q., and Shek, K.-L., 1991, "Analysis of Cracked Plates With a Bonded Patch,” Eng. Fract. Mech., 40, No. 6, pp. 1055-1065.

[9] Karlsson, A. M., and Bottega, W. J., 2000, "On the Behavior of a Class of Patched Plates During Cooling," Int. J. Non-Linear Mech., 35, No. 3, pp. $353-566$.

[10] Karlsson, A. M., and Bottega, W. J., 2000, “'On Thermal Buckling of Patched
Beam-Plates,” Int. J. Solids Struct., 37, No. 34, pp. 4655-4690.

[11] Karlsson, A. M., and Bottega, W. J., 2000, “'On the Thermo-Mechanical Response of Patched Plates," AIAA J., 38, No. 6, pp. 1055-1062.

[12] Bottega, W. J., 1995, "Separation Failure In a Class of Bonded Plates,' Compos. Struct., 30, No. 3, pp. 253-269.

[13] Bottega, W. J., and Karlsson, A. M., 1999, "On the Detachment of StepTapered Doublers: Part 1-Foundations,'” Int. J. Solids Struct., 36, No. 11, pp. $1597-1623$

[14] Karlsson, A. M., and Bottega, W. J., 1999, “The Presence on Edge Contact and its Influence on the Debonding of Patched Panels,' Int. J. Fract., 96, No. 4, pp. 383-406.

[15] Karlsson, A. M., and Bottega, W. J., 1996, “On the Detachment of StepTapered Doublers: Part 2-Evolution of Pressure Loaded Structures,' Int. J. Solids Struct., 36, No. 11, pp. 1626-1651

[16] Loia, M. A., and Bottega, W. J., 1995, “'On Planar-Tensile Representation of Edge Debonding in Patched Panels Under Pressure,' Int. J. Solids Struct., 15, No. 4, pp. 211-217.

[17] Hutchinson, J. W., and Suo, S., 1992, "Mixed Mode Cracking in Layered Materials," Adv. Appl. Mech., 29, pp. 63-191. 